\title{
LA COLECCIÓN HISTÓRICA DE ESPONJAS DEL MUSEO NACIONAL DE CIENCIAS NATURALES (MNCN.CSIC): 1771-1939
}

\author{
J. I. Sánchez-Almazán \\ Museo Nacional de Ciencias Naturales. C.S.I.C. c/José Gutiérrez Abascal, 2. 28006. Madrid. España. \\ ORCID iD (PC): http://orcid.org/0000-0001-9489-3724. E-mail: jsalmazan@mncn.csic.es
}

\section{RESUMEN}

En esta publicación se presenta por vez primera un estudio de la colección histórica de esponjas del Museo Nacional de Ciencias Naturales, que reúne material procedente de tres períodos: la época del Real Gabinete de Historia Natural (1771 a 1815), el último cuarto del siglo XIX y los años que van de 1901 a 1939, en los que fue director Ignacio Bolívar. La colección comprende más de 800 ejemplares, de 143 especies. De la primera época se conservan 61, de 26 especies: 41 de la colección de Pedro Franco Dávila o de los años en que éste fue director del Real Gabinete (1771-1786), 13 de Antonio Parra, seis de Rojas Clemente y uno de la expedición Malaspina (1789-1794). Otros 350, en su mayoría preparaciones microscópicas, de 40 especies, son de fines del XIX. Por último, más de 400 (120 especies) ingresaron bajo la dirección de lgnacio Bolívar (1901-1939). Este trabajo tiene un triple objetivo: 1) Continuar el estudio del material procedente del Real Gabinete, iniciado en el período 2003-2007; 2) presentar los resultados del trabajo de recuperación, realizado desde 2014, de más de 400 preparaciones microscópicas de esponjas del siglo XIX y comienzos del XX que estaban sin inventariar, junto con una revisión del resto de material de esa época; 3 ) investigar la obra de Francisco Ferrer Hernández, que destacó en la época de Bolívar identificando más de 280 ejemplares y describiendo una docena de especies nuevas.

Palabras clave: Esponjas; Colección histórica; Real Gabinete de Historia Natural; Pedro Franco Dávila; Antonio Parra; Simón de Rojas Clemente; Francisco Ferrer Hernández; Ignacio Bolívar.

\section{ABSTRACT}

The historical sponges Collection in the Museo Nacional de Ciencias Naturales (MNCN.CSIC): 1771-1939

A study of the collection of historical sponges of the Museo Nacional de Ciencias Naturales is presented. This collection contains material from three different periods of the institution: the era of the Royal Cabinet of Natural History (1771-1815), the late XIX and the first third of XX century, under the direction of Ignacio Bolívar. There are over 800 specimens (143 species) of which 61 (26 species) come from the first period: 41 directly from Pedro Franco Dávila Collection or the years when Dávila was director of the Royal Cabinet (1771-1786), 13 from Antonio Parra Collection, six from Rojas Clemente and one from the Malaspina Expedition (1789-1794). The Museum preserves 350 pieces, mostly microscopic slides (over 40 species), from the late nineteenth century. Finally, more than 400 (120 species) entered along the period of Ignacio Bolivar as director of the Museum (1901-1939). This work has a threefold objective: 1 . To continue the study of the material from the Royal Cabinet, which was commenced during 2003-2007. 2. To present the results of the recovery of more than 400 microscopic slides from late nineteenth and the beginning of XX, a work that commenced in 2014 , together with a review of the rest of the material of this period. 3 . To investigate the work of Francisco Ferrer Hernández, who highlighted during Bolívar period. Ferrer identified more of 280 specimens and described a dozen of new species.

Key words: Sponges; Historical collection; Royal Cabinet of Natural History; Pedro Franco Dávila; Antonio Parra; Simón de Rojas Clemente; Francisco Ferrer Hernández; Ignacio Bolívar.

Cómo citar este artículo/Citation: Sánchez-Almazán, J. I. 2017. La colección histórica de esponjas del Museo Nacional de Ciencias Naturales (MNCN.CSIC): 1771-1939. Graellsia, 73(2): e060. http://dx.doi.org/10.3989/graellsia.2017.v73.181

Copyright: (C) 2017 SAM y CSIC. This is an open-access article distributed under the terms of the Creative Commons Attribution (CC-by) Spain 3.0 License. 


\section{Introducción}

La colección de esponjas (filum Porifera) del Museo Nacional de Ciencias Naturales $(\mathrm{MNCN})$ reúne más de 1.500 ejemplares de 26 órdenes, 60 familias y más de 250 especies diferentes. La mayoría son del Mediterráneo (alrededor del $50 \%$ de los ejemplares) y del Atlántico (un $20 \%$ ) y un pequeño número proviene del Pacífico y del Océano Glacial Antártico. Incluye 86 tipos, 69 de ellos holotipos.

Algo más de la mitad de los ejemplares, en número superior a los 800 , son históricos. Se consideran como tales aquéllos cuyo ingreso se realizó antes de 1940. Dichos ejemplares constituyen el objeto del presente trabajo. Este material procede de tres de los períodos más brillantes de la historia de la institución: su primera etapa, de 1771 a 1815 , como Real Gabinete de Historia Natural (RGHN), el último cuarto del siglo XIX (cuando cobraron auge los estudios de organismos marinos) y el período de Ignacio Bolívar (1901-1939). En cada uno de estos períodos destacó la labor de un naturalista que con su trabajo contribuyó al conocimiento de estos organismos: Antonio Parra en tiempos del RGHN, Augusto González de Linares a finales del siglo XIX y Francisco Ferrer Hernández en la época de Bolívar.

\section{LA ÉPOCA DEL REAL GABINETE DE HISTORIA NATURAL (1771-1815)}

El Real Gabinete de Historia Natural (RGHN), creado en Madrid en 1771, tuvo como primer director a Pedro Franco Dávila (1711-1786), ilustrado guayaquileño que había reunido en París un afamado gabinete de historia natural. Con sus colecciones se formaron los fondos del RGHN, una parte importante de los cuales estaban integrados por invertebrados marinos, descritos en el volumen primero de su Catalogue Systématique et Raisonné des Curiosités de la Nature et de l'Art qui composent le Cabinet de Mr. Davila (Franco-Dávila, 1767). Son unas 80 las esponjas descritas por Dávila en su libro, que figuraban en el Orden Segundo de los Poliparios, en el Género $5^{\circ}$ («Esponjas»), más otra media docena descritas en el Género $6^{\circ}$ («Alciones»). En esa época algunos autores aún dudaban de la verdadera naturaleza de las esponjas y sostenían que eran vegetales, no así Dávila, que en su obra decía: «Las cavidades que forman son probablemente la obra y la morada de una cierta clase de animálculos todavía poco conocidos». Resulta difícil determinar cuántos de esos ejemplares del gabinete que Dávila formó en París se han conservado en la actual colección, ya que en la mayoría de las descripciones del Catalogue no se dan detalles suficientes como para identificar las piezas con seguridad. Sin embargo, sí se han datado diversos ejemplares de esponjas procedentes del RGHN, cuya cuantificación se aborda en el apartado de «Material y Métodos».
Otras dos colecciones nutrieron al RGHN de esponjas tras el fallecimiento de Dávila: la de Antonio Parra y la de Rojas Clemente. Hay también un ejemplar de la expedición Malaspina (1789-1794).

La llegada de la Colección Parra al RGHN fue consecuencia de la política de obtención de «producciones naturales» seguida por Dávila. En 1776 Dávila redactó por orden del rey el borrador de una Instrucción dirigida a todas las autoridades del imperio para que enviaran al RGHN cuantas «producciones curiosas de la Naturaleza se hallasen en sus dominios». Ello produjo la entrada de numerosas piezas, lo que se completó con la petición de ejemplares a los principales museos y sociedades científicas de Europa, gracias a las relaciones establecidas por Dávila en París con prestigiosos naturalistas de la época (SánchezAlmazán, 2012). Otras entradas se debieron a intercambios y a los envíos de corresponsales facultados por toda la península Ibérica.

Antonio Parra, de cuya vida se tienen pocos datos (se desconoce, por ejemplo, la fecha de su muerte), era un portugués nacido en 1739 en Tavira y establecido en La Habana alrededor de 1763 que había iniciado una colección de «producciones naturales» de Cuba. Dávila le envió una carta el 3 de septiembre de 1778, de la que el destinatario acusó recibo en su contestación desde La Habana en marzo de 1779. El interés inicial de Parra era la ictiofauna pero, a instancias de Dávila, comenzó a recolectar también crustáceos decápodos, esponjas y corales. Por dos veces intentó Parra viajar a España, en 1779 y en 1786, pero sus tentativas resultaron fallidas. En 1787 publicó Descripción de diferentes piezas de Historia Natural las más del ramo marítimo representadas en setenta y cinco láminas (Parra, 1787) que tuvo gran aceptación (Fig. 1). No será hasta 1789 cuando, por fin, realice su viaje a nuestro país (Dávila había fallecido en 1786) y sus colecciones ingresen en el RGHN. En su libro Parra incluía las esponjas en la «Parte Segunda. Plantas Marítimas».

Hay que señalar también las especies recogidas por Simón de Rojas Clemente y Rubio (1777-1827). Este naturalista estudió botánica con destacados científicos, como Casimiro Gómez Ortega (1741-1818) y Antonio José de Cavanilles (1745-1804). En 1802 acompañó a Inglaterra y Francia a Domingo Badía (1767-1818), al que le unió una fuerte amistad. Badía fue un famoso militar y aventurero español más conocido como Alí Bey, nombre que adoptó como parte de su caracterización como príncipe musulmán de origen andalusí para cumplir una misión de espionaje, encargada por Godoy, que le llevó en 1803 por tierras del norte de África y del imperio otomano. Rojas Clemente, que se había preparado para ir con Badía a África, no llegó a realizar el viaje, en un principio concebido con un interés estrictamente científico. Entre 1803 y 1804 recorrió la costa gaditana reuniendo una colección de algas y otras «producciones marinas», que llevó al 


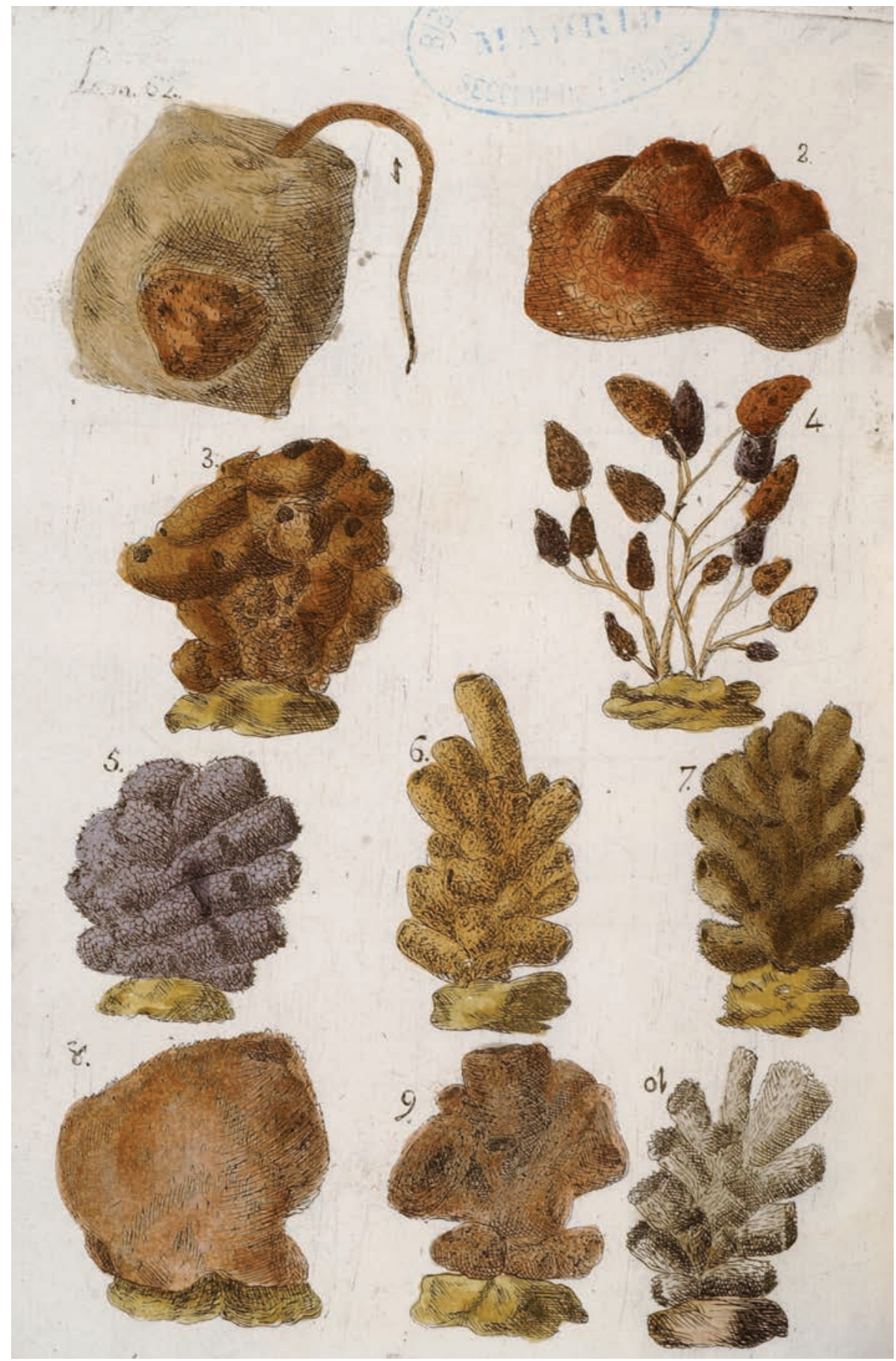

Fig. 1.- Lámina 62 del libro de Antonio Parra. Figuras de esponjas realizadas por su hijo Manuel Antonio Romualdo. Se identifican con claridad ocho esponjas históricas de la colección actual (Jesús Muñoz. Servicio de Fotografía del MNCN).

Fig. 1.- Plate 62 from the book of Antonio Parra. Sponges drawings executed by his son Manuel Antonio Romualdo. Eight historical sponges of the current collection are identified from this plate (Jesús Muñoz. MNCN Photography Service).

Real Jardín Botánico cuando en 1805 fue llamado a éste para hacerse cargo de la biblioteca.

En 2001 el Real Jardín Botánico se puso en contacto con la Colección de Invertebrados del MNCN para entregar 27 muestras, preparadas como un herbario, que había custodiado durante casi 200 años. Las muestras habían sido revisadas por Javier Cremades, como parte del estudio para su tesis doctoral sobre el herbario de plantas marinas de Rojas Clemente (Cremades, 1990, 1993). Se trataba de organismos animales (siete poríferos, seis briozoos y 14 hidrozoos), todos colectados por Rojas Clemente entre 1803 y 1804, en su mayoría en Sanlúcar de Barrameda, excepto uno sin identificar procedente de la expedición Malaspina, recogido por Luis Née (1734-1803). 


\section{FINALES DEL SIGLO XIX}

Dentro de la azarosa historia del Museo de Ciencias en el siglo XIX, en el último tercio de éste hubo importantes innovaciones para las colecciones de invertebrados. En 1875 Ignacio Bolívar y Urrutia (1850-1944) fue nombrado ayudante de Zoología. Dos años más tarde pasaría a ocupar la Cátedra de Animales Articulados -artrópodos- y Lucas de Tornos, entonces director del centro, la de Malacología y Animales Inferiores. Por esa época el museo estaba ligado a la enseñanza universitaria, vinculación que continuaría durante el primer tercio del siglo XX. Estrecha relación con el museo tuvo la Estación Marítima de Zoología y Botánica Experimentales -más conocida como Estación de Biología Marina de Santander-, creada en 1886 por las gestiones de Augusto González de Linares (1845-1904) y Bolívar. Este organismo, pionero en España de los estudios marinos, estuvo adscrito al Museo de Ciencias de 1901 a 1914. Por esos años se firmaron convenios de colaboración con centros extranjeros, como la Estación de Biología Marina de Nápoles, creada en 1874. De las estaciones de Santander y Nápoles llegarían al museo diversos ejemplares de esponjas. Tras el polémico traslado del museo en 1895 de su sede de la calle de Alcalá al Palacio de Bibliotecas y Museos (actual Biblioteca Nacional), en 1901 fue nombrado director Ignacio Bolívar, quien defendió la necesidad de un nuevo edificio para el centro. En 1907 se concedió el Pabellón norte del Palacio Nacional de Exposiciones de las Artes y la Industria, donde hoy se encuentra. Más tarde se ocuparía también el Pabellón sur.

\section{LA ÉPOCA DE IGNACIO BOLÍIAR (1901-1939)}

Francisco Ferrer Hernández, del que apenas se tienen datos biográficos, trabajó en el Museo de Ciencias durante la época de Bolívar y fue un destacado especialista en esponjas, grupo sobre el que realizó diversas publicaciones, aparecidas en el Boletín de la Real Sociedad Española de Historia Natural y en la serie Trabajos del Museo Nacional de Ciencias Naturales (Ferrer-Hernández, 1912，1914a，1914b，1914c, 1916, 1917, 1918, 1919, 1921, 1932a, 1932b, 1933). Nombrado en 1913 auxiliar numerario del tercer grupo de la Sección de Naturales de la Facultad de Ciencias de la Universidad Central ${ }^{1}$, estudió la fauna de esponjas del Cantábrico, así como de las Baleares.

Ferrer Hernández realizó capturas de esponjas en diferentes puntos del litoral ibérico: en 1912 y 1914, en Santander; en el invierno de 1914-1915 en Baleares, Mahón y Menorca; en 1918 en Asturias; en agosto y septiembre de 1921 en la bahía de Palma de Mallorca y los puertos de Mahón y Cabrera (FerrerHernández, 1921) y en 1933 en los alrededores de

1. El Heraldo de Madrid, 10 de noviembre.
Marín (Ferrer-Hernández, 1933). Sus estudios sobre las esponjas del Cantábrico dieron como resultado dos volúmenes, publicados en Trabajos del Museo Nacional de Ciencias Naturales (Ferrer-Hernández, $1914 b, 1914 c)$. Además revisó la colección de preparaciones del geólogo Domingo de Orueta (1862-1926) (Ferrer-Hernández, 1918) y describió otras muchas especies: varias recogidas en Málaga por Enrique Rioja Lo Bianco (1895-1963) (Ferrer-Hernández, 1917); otras de Castellón de la Plana enviadas por Antimo Boscá (1874-1950) (Ferrer-Hernández, 1919); una del género Acanthascus, traída por Orueta de Filipinas (Ferrer-Hernández, 1932b), y otra de la clase Hexactinellida que consideró especie nueva y denominó Corythophora ijimai (Ferrer-Hernández, 1932a), hoy Sericolophus reflexus (Ijima, 1894).

En el verano de 1933 Ferrer Hernández participó en una serie de conferencias organizadas por la Junta para la Ampliación de Estudios e Investigaciones Científicas (JAE) impartidas para alumnos pensionados en la Estación de Biología de Marín, creada en 1932 y dependiente del museo, pues la estación de Santander había pasado a pertenecer al Instituto Español de Oceanografía en 1914, tras la creación de éste. Las conferencias impartidas por Ferrer fueron tres $^{2}$ : « $1^{\mathrm{a}}$. Generalidades sobre el plancton marino y problemas que se plantean al estudiar el plancton de la ría de Marín; 2a . Distribución, recolección, preparación y clasificación de los Espongiarios; $3^{\mathrm{a}}$. Distribución, recolección, conservación y clasificación de los Equinodermos». En 1934 Ferrer Hernández intervino en un congreso celebrado en Rabat con la memoria «Considérations sur les caracteres et la répartition géographique de quelques éponges de Majorque ${ }^{3}$ ). En el Apéndice 2 se transcribe una breve carta de Ferrer, del 23 de abril de $1932^{4}$, donde da cuenta de su labor investigadora, tal y como había solicitado la JAE, en instrucciones emitidas para la fiscalización del personal investigador a su cargo «con el fin de elaborar las nóminas, conocer las necesidades y controlar el cumplimiento del trabajo».

Los principales colectores de esponjas con destino al museo en esta época fueron, además del propio Ferrer: José Rioja, González de Linares, Gómez Llueca, Fernando de Buen y Lozano, Odón de Buen, Enrique Rioja y Domingo de Orueta. José Rioja Martín (1866-1945), padre de Enrique Rioja lo Bianco, fue director de la Estación de Santander: en

2. Documento ACN0373/005 del Archivo del MNCN. Fondo Museo. Dirección. Serie Expedientes informativos. Año 1933.

3. Documento ACN0306/013 del Archivo del MNCN. Fondo Museo. Exposiciones y Programas Públicos. Serie Congresos Internacionales. Año 1934. Carta dirigida desde Tetuán a Ignacio Bolívar del 24 de marzo de ese año.

4. Documento ACN0352/007/001 del Archivo del MNCN. Fondo Junta para Ampliación de Estudios e Investigaciones Científicas. Serie Expedientes de gestión administrativa. Año 1932. 
la colección hay 68 ejemplares recogidos por él en Pontevedra, en los veranos de 1932 y 1933 . Augusto González de Linares recogió casi una veintena de esponjas, en los primeros años del siglo, la mayoría de Santander, entre las cuales había dos especies descritas por Ferrer como especies nuevas: Erylus cantabricus y Cantabrina erecta. El paleontólogo Federico Gómez Llueca (1889-1960) colectó 10 ejemplares de Palma de Mallorca. Del ictiólogo Fernando de Buen y Lozano (1895-1962) hay 15 ejemplares de Mallorca, recogidos entre 1932 y 1934. Un pequeño número de esponjas fueron traídas por otros notables científicos: casi una decena, sobre todo de Baleares, proceden de Odón de Buen (1863-1945), pionero de la oceanografía en España y fundador del Instituto Español de Oceanografía; 12 son de Enrique Rioja Lo Bianco, varias ingresadas antes de 1917, procedentes de Málaga; siete de Orueta, de Gijón, y cuatro preparaciones microscópicas de Antonio de Zulueta (18851971), pionero de la genética en España, ingresadas en torno a 1906. Dos especies de esponjas llevan el nombre de Ferrer Hernández como reconocimiento a su labor: Protosuberites ferrerhernandezi (BouryEnault \& Lopes, 1985) y Phorbas ferrerhernandezi van Soest, 2002. En 1910 le fue dedicado un género, Ferrerhernandezia, en la especie Ferrerhernandezia aenigma (Lundbeck, 1910), en la actualidad sin validez, pues dicha especie se ha renombrado como Acanthacora aenigma (Lundbeck, 1910).

El presente trabajo persigue un triple objetivo: 1) Proseguir el estudio del material procedente del Real Gabinete, iniciado entre 2003 y 2007; 2) presentar los resultados del trabajo de recuperación, realizado desde 2014, de más de 400 preparaciones microscópicas de esponjas del siglo XIX y comienzos del XX que estaban sin inventariar, junto con una revisión del resto de material de esa época; y 3 ) investigar la obra de Francisco Ferrer Hernández, cuyo trabajo se desarrolló en la época de Bolívar, fruto del cual fue la identificación de más de 280 ejemplares y la descripción de una docena de especies nuevas.

\section{Material y métodos}

A finales de los años 80 del siglo pasado se procedió a la revisión del material existente en la colección de esponjas del MNCN, la cual, como ocurrió con otras partes de los fondos del museo, había permanecido durante casi 40 años prácticamente en estado de abandono. De los poco más de 500 ejemplares inventariados, 54 se identificaron como material procedente de la época del Real Gabinete. Se trataba de ejemplares en seco, varios de grandes dimensiones, la tercera parte con peanas de madera, 15 de éstas imitando una rocalla de tipo rococó. Algunas esponjas tenían asignados números en etiquetas redondas con arandela dorada, similares a los de otras colecciones, procedentes de un catálogo antiguo que no ha podido encontrarse.
Entre 2003 y 2007 se abordó el estudio e identificación del material perteneciente a la colección Parra (Villena et al., 2009), 13 ejemplares que aparecían descritos y dibujados en la obra que el naturalista publicó en 1787, la cual se revisó exhaustivamente, así como la carta de Parra a Dávila del 12 de marzo de $1779^{5}$. También se examinaron dos ejemplares enviados por el naturalista ginebrino Jean André De Luc (1727-1817). En el archivo del MNCN existen dos cartas cruzadas entre Dávila y De Luc. La primera es del 21 de junio de 1765, dirigida por Dávila a De Luc solicitándole varias piezas, al tiempo que le envía algunos ejemplares de los "duplicados" existentes en el RGHN. La segunda, del 5 de agosto, es la respuesta de De Luc, en la que anuncia el envío de una caja con ejemplares junto a un catálogo de 34 esponjas ${ }^{6}$. Dicho estudio se completó en 2014 con la revisión del resto del material procedente del Real Gabinete (41 piezas), 20 de cuyos ejemplares, junto con los de Parra, habían sido examinados por análisis de espículas en 2003 por Rob W. M. van Soest y Dirk Erpenbeck: 10 se identificaron al nivel de género y 21 al de especie (tres con dudas). Todas las especies asignadas a la Colección Parra correspondían a especies propias del Caribe, como cabía esperar.

También se han estudiado recientemente las muestras entregadas en 2001 por el Real Jardín Botánico (seis piezas procedentes de Rojas Clemente y una de la expedición de Malaspina). Estas últimas habían sido revisadas por Javier Cremades en su tesis doctoral (Cremades, 1990). De las esponjas pertenecientes a la época del Real Gabinete, las únicas etiquetas originales que se han conservado son las correspondientes a estas últimas muestras.

En cuanto al material procedente de finales del siglo XIX, salvo un $10 \%$, que está conservado en alcohol, el resto, consistente en preparaciones microscópicas, estaba sin inventariar y se ha estudiado entre 2014 y 2016. La mayoría de dichas preparaciones conservan una etiqueta pegada al portaobjetos, pero en muchas de ellas faltan datos esenciales, como la fecha de preparación y/o captura del ejemplar, la localidad o la persona que hizo la determinación. En 55 de ellas sólo hay escritas unas siglas, cifras y medidas (en micras). Sin embargo, el tipo de etiqueta, la caligrafía y los datos numéricos anotados, similares a las correspondientes al siglo XIX, indican que pueden ser de esa época, por lo que se han asignado a ella con dudas. El dato de las personas que identificaron los ejemplares sólo aparece en una veintena de piezas y en su mayoría corresponde a Augusto González Linares y Francisco Ferrer Hernández.

Respecto al material de la época de Bolívar, se han inventariado en los dos últimos años casi un

\footnotetext{
5. Calatayud-Arinero, 1987: CDRGHN (1752-1786). Ref. 548. Archivo del MNCN.

6. Calatayud-Arinero, 1987: CDRGHN (1752-1786). Ref. 60. Archivo del MNCN.
} 
centenar de preparaciones microscópicas de ese período, lo que supone una cuarta parte del material procedente de esos años. El resto, conservado en alcohol, estaba inventariado pero no estudiado. El autor de la determinación de los ejemplares figura en las dos terceras partes de éstos y en ese cometido destacó Francisco Ferrer Hernández, que determinó el $65 \%$ de ellos. Intervinieron también, aunque en mucha menor medida, Augusto González Linares, Enrique Rioja, José Rioja, Odón de Buen y Domingo de Orueta.

Los datos relativos a la localidad, fecha de captura (cuando existe), colector o donante y persona que determinó el ejemplar en el material en alcohol, tanto de finales del siglo XIX como de la época de Bolívar, proceden del Inventario de madréporas, celentéreos y esponjas del Museo de Historia Natural, realizado en $1945^{7}$ y estudiado en 2001 por Miguel Villena, conservador de la Colección de Invertebrados desde 2001 a 2006.

Una parte importante del presente trabajo ha consistido en estudiar las aportaciones de Ferrer Hernández, por lo cual se han revisado sus publicaciones y también los documentos que se conservan en el Archivo del MNCN relacionados con este naturalista y que atestiguan su labor en el museo. Estos documentos, ya mencionados en el apartado anterior, son: ACN0373/005 Fondo Museo. Dirección. Serie Expedientes informativos. Año 1933; ACN0306/013 Fondo Museo. Exposiciones y Programas Públicos. Serie Congresos Internacionales. Año 1934; ACN0352/007/001 Fondo Junta para Ampliación de Estudios e Investigaciones Científicas. Serie Expedientes de gestión administrativa. Año 1932. A ellos hay que añadir uno más, ACN0275/028 MNCN Fondo Museo. Colecciones. Ingresos y salidas. Año 1915. En él se consigna una donación de Ferrer al centro de más de un centenar de esponjas.

La actualización de la nomenclatura científica de la colección histórica de esponjas se ha realizado de acuerdo con la World Porifera Database (van Soest et al., 2017), incluida en la base de datos World Register of Marine Species (WoRMS Editorial Board, 2017).

\section{Resultados}

En la colección histórica de esponjas del MNCN se conservan más de 800 ejemplares pertenecientes a 24 órdenes, 56 familias y 143 especies (Apéndice 1). Las familias con mayor número de ejemplares son: Geodiidae ( $>190)$, Halichondriidae $(>80)$, Chalinidae, Ancorinidae, Axinellidae y Suberitidae $(\sim 40)$, Grantiidae $(>30)$ y Spongiidae $(\sim 25)$. De estos ejemplares, 61 proceden de la época del Real Gabinete de Historia Natural (1771-1815), 350 del último cuarto del siglo XIX y casi 400 del período en el que Ignacio Bolívar fue director (1901-1939). Todos los recuentos

7. Documento ACN0246/006. Archivo del MNCN. efectuados, que se ofrecen en este apartado, proceden de la información contenida en la base de datos de la Colección de Invertebrados.

\section{ESPONJAS DE LA ÉPOCA DEL REAL GABINETE DE HISTORIA NATURAL (1771-1815)}

Actualmente se estiman en 61 los ejemplares de esponjas del MNCN que pertenecieron al Real Gabinete, bien por proceder de la colección creada por Dávila en París o por ingresar cuando él era director de la institución, bien por haberse colectado en esa época aunque no ingresaran en su momento en el Real Gabinete (como el material entregado por el Real Jardín Botánico al MNCN en 2001): 26 se han identificado al nivel de especie y otros ocho al de género (Tabla 1). Sólo en 18 casos puede documentarse la localidad, ya sea por haberse conservado la etiqueta original (material procedente del Real Jardín Botánico), por existir documentos históricos que lo atestiguan (los dos ejemplares enviados por Jean André De Luc) o por haberse identificado los ejemplares como especies características de un área geográfica dada (caso de las esponjas de Parra).

Entre los posibles ejemplares procedentes de la colección formada por Dávila destacan:

MNCN 1.01/16. Spongia sp. Tiene forma de embudo. Superficie blanda, esponjosa, muy porosa. Color pardo amarillento. Altura: $28 \mathrm{~cm}$.

MNCN 1.01/21. Posible Hyattella cavernosa (Pallas, 1766). Peana rococó, similar a las de Parra. Forma ramificada, compacta. Colonias de aspecto piloso. Altura: $30 \mathrm{~cm}$ (Fig. 2).

MNCN 1.01/33. Callyspongia vaginalis (Lamarck, 1814). Peana de madera negra torneada. Se compone de siete túbulos altos, alargados y ramificados. Altura: unos $30 \mathrm{~cm}$.

MNCN 1.01/315. Agelas sceptrum (Lamarck, 1815). Ramificada, de ramas aplanadas y color pardo amarillento. Peana torneada negra. Altura: unos $50 \mathrm{~cm}$.

MNCN 1.01/317. Axinella polypoides Schmidt, 1862. Varios túbulos delgados, ramificados y de color pardo claro. Altura: $50 \mathrm{~cm}$.

Un ejemplar de Cliona patera (Hardwicke, 1820), MNCN 1.01/38, con forma de copa, rojiza y de aspecto reticulado, de unos $40 \mathrm{~cm}$ de altura. Tiene una peana rococó con organismos marinos incrustados, semejante también a la de las piezas de Parra.

Hay además otros dos ejemplares de Cliona patera de gran tamaño: MNCN 1.01/36 (de $80 \mathrm{~cm}$ de altura y un diámetro del ósculo de unos $50 \mathrm{~cm}$ ) y MNCN $1.01 / 316$ (de $45 \mathrm{~cm}$ ), con forma de copa, de color pardo claro, enviadas probablemente por Jean André De Luc.

Se han identificado 17 piezas de la actual Colección de Invertebrados procedentes de Parra: 13 esponjas y cuatro corales (Villena et al., 2009). Varios de los ejemplares de esponjas sobresalen por su carácter singular: 
Tabla 1.- Esponjas históricas procedentes de la época del RGHN.

Table 1.- Historical sponges from the time of RGHN.

\begin{tabular}{|c|c|c|}
\hline COLECCIÓN & ESPECIES & EJEMPLARES DE COLECCIÓN \\
\hline $\begin{array}{l}\text { DÁVILA } \\
\text { o anterior a } 1786\end{array}$ & $\begin{array}{l}\text { Agelas conifera } \\
\text { Agelas dispar } \\
\text { Agelas sceptrum } \\
\text { Aplysina archeri } \\
\text { Aplysina fistularis } \\
\text { Axinella polypoides } \\
\text { Callyspongia fallax } \\
\text { Callyspongia plicifera } \\
\text { Callyspongia vaginalis } \\
\text { Clathria sp. } \\
\text { Clathrina spinosa } \\
\text { Cliona celata } \\
\text { Cliona patera } \\
\text { Echinodictyum pennatum } \\
\text { Forcepia sp. } \\
\text { Hippospongia sp. } \\
\text { Hyattella cavernosa ¿? } \\
\text { Niphates erecta } \\
\text { Raspailia sp. } \\
\text { Scalarispongia sp. } \\
\text { Spongia sp. } \\
\text { Taonura byssoides } \\
\text { Sin determinar }\end{array}$ & $\begin{array}{l}M N C N 1.01 / 341 \\
M N C N 1.01 / 334 \\
M N C N 1.01 / 315 \\
M N C N 1.01 / 318 \text { y } 340 \\
M N C N 1.01 / 37 \\
M N C N 1.01 / 317 \\
M N C N 1.01 / 331 \\
M N C N 1.01 / 336 \\
M N C N 1.01 / 33 \\
M N C N 1.01 / 328 \text { y } 333 \\
M N C N 1.01 / 343 \\
M N C N 1.01 / 321 \\
M N C N 1.01 / 36,38 \text { y } 316 \\
M N C N 1.01 / 344 \\
M N C N 1.01 / 327 \\
M N C N 1.01 / 35 \\
M N C N 1.01 / 21 \\
M N C N 1.01 / 338 \\
M N C N 1.01 / 24 \text { y } 335 \\
M N C N 1.01 / 337 \text { y } 342 \\
M N C N 1.01 / 16 \text { y } 18 \\
M N C N 1.01 / 31 \\
M N C N 1.00 / 1,2,5,17,34,39,54,58 \text { y } 66 \\
M N C N 1.01 / 17,20 \text { y } 22\end{array}$ \\
\hline $\begin{array}{l}\text { COLECCIÓN } \\
\text { PARRA }\end{array}$ & $\begin{array}{l}\text { Aplysina lacunosa } \\
\text { Callyspongia plicifera } \\
\text { Callyspongia tenerrima } \\
\text { Callyspongia vaginalis } \\
\text { Gelliodes sp. } \\
\text { Hippospongia lachne } \\
\text { Leiosella sp. } \\
\text { Mycale mirabilis } \\
\text { Smenospongia conulosa } \\
\text { Spongia officinalis } \\
\text { Spongia sp. } \\
\text { Xestospongia muta }\end{array}$ & $\begin{array}{l}\text { MNCN 1.01/325 } \\
\text { MNCN 1.01/313 } \\
M N C N 1.01 / 314 \\
\text { MNCN 1.01/312 } \\
\text { MNCN 1.01/319 } \\
\text { MNCN 1.01/19 } \\
M N C N 1.01 / 332 \\
M N C N 1.01 / 326 \\
M N C N 1.01 / 323 \\
M N C N 1.01 / 32 \\
M N C N 1.01 / 28 \text { y } 320 \\
M N C N 1.01 / 324\end{array}$ \\
\hline COLECCIÓN ROJAS CLEMENTE & $\begin{array}{l}\text { Halichondria panicea } \\
\text { Haliclona oculata } \\
\text { Spongia officinalis }\end{array}$ & $\begin{array}{l}\text { MNCN 1.01/698, } 699 \text { y } 701 \\
M N C N 1.01 / 700 \\
\text { MNCN 1.01/303 y } 304\end{array}$ \\
\hline EXPEDICIÓN MALASPINA & Sin determinar & MNCN 1.00/133 \\
\hline
\end{tabular}

MNCN 1.01/28. Spongia sp. Colonias con forma de embudo. Color pardo claro. Altura: $50 \mathrm{~cm}$. Ejemplar $\mathrm{n}^{\circ} 9$ de la lámina 62 de la obra de Parra, del que se dice: «se parece cada una à una copa, y aunque es mole [blanda] en algún modo, especialmente acabada de coger. Su texido es bien compacto. No tiene células ni conductos...».

MNCN 1.01/32. Posible Spongia officinalis Linnaeus, 1759. Colonias tubulares o con forma de embudo. Altura: $50 \mathrm{~cm}$. Ejemplar $\mathrm{n}^{\circ} 6$ de la lámina 62 de la obra de Parra.

MNCN 1.01/312. Callyspongia vaginalis (Lamarck, 1814). Colonias tubulares con nerviaciones en las paredes. Superficie externa de aspecto espinoso. Altura: 60 $\mathrm{cm}$. Ejemplar n $\mathrm{n}^{\circ} 11$ de la lámina 63 de la obra de Parra. De ella dice el autor: «La Figura 11 representa las flautas de un órgano; su color es obscuro, es muy flexible.
En su interior manifiesta unas líneas que vienen del fondo al borde, y en su exterior unos granitos mas ó menos finos»».

MNCN 1.01/313. Callyspongia plicifera (Lamarck, 1814). Colonias con forma de barriletes, acampanadas o con aspecto de embudo las más grandes. Superficie surcada por pliegues profundos. Altura: $45 \mathrm{~cm}$. Ejemplar $n^{\circ} 12$ de la lámina 63 en la obra de Parra.

MNCN 1.01/319. Gelliodes sp. Colonias alargadas, más o menos tubulares, aplanadas y ramificadas, con la superficie finamente reticulada. Color pardo rojizo. Altura: $40 \mathrm{~cm}$. Ejemplar n ${ }^{\circ} 20$ de la lámina 63 de la obra de Parra donde se dice: «agradabilísima por su color carmesí, tiene diversas figuras, pues cada una hace una obra diferente: parece su obra exterior al terciopelo de algodón, solo con la diferencia de tener algunos agujeros». 


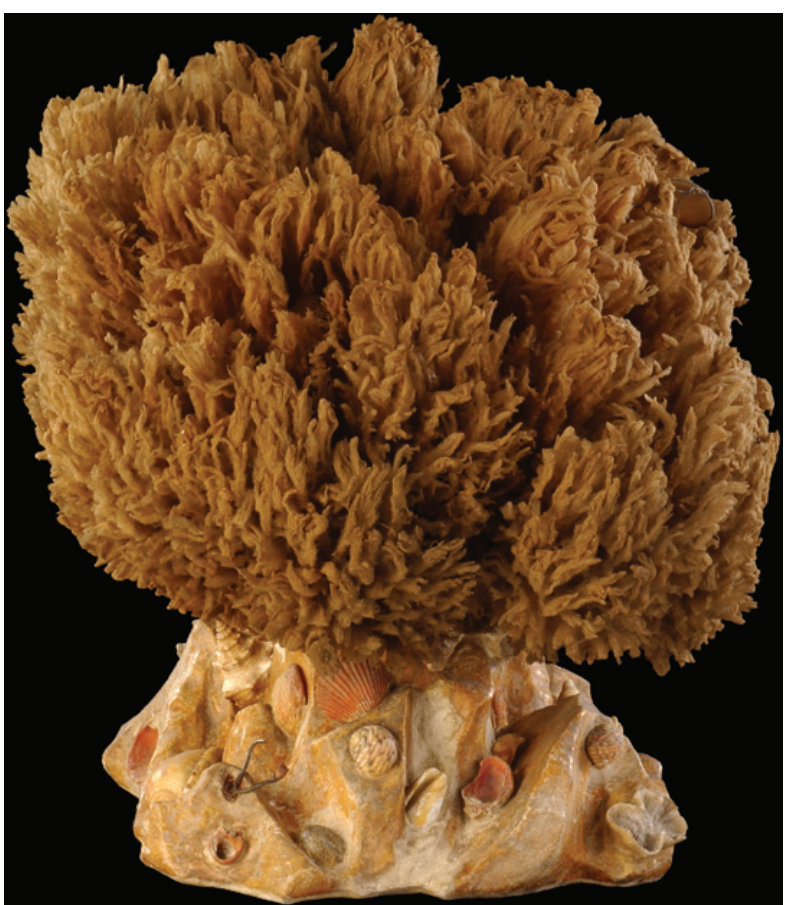

Fig. 2.- Hyatella cavernosa (Pallas, 1766): MNCN 1.01/21. Uno de los ejemplares históricos de esponjas procedentes del RGHN (Jesús Muñoz. Servicio de Fotografía del MNCN).

Fig. 2.- Hyatella cavernosa (Pallas, 1766): MNCN 1.01/21. Historical sponges collection from RGHN (Jesús Muñoz. MNCN Photography Service).

MNCN 1.01/323. Smenospongia conulosa Pulitzer-Finali, 1986. Colonias compactas, las mayores con forma de pequeños odres. Superficie reticulada. Color pardo claro. Altura: $40 \mathrm{~cm}$. Ejemplar $\mathrm{n}^{\circ} 3$ de la lámina 62 de la obra de Parra (Fig. 3).

MNCN 1.01/324. Xestospongia muta (Schmidt, 1870). Forma de barril con un gran ósculo. Altura: $40 \mathrm{~cm}$. Ejemplar $\mathrm{n}^{\circ} 1$ de la lámina 62 de la obra de Parra, del que se proporciona una completa descripción: «La primera que se presenta, que manifiesta la figura de un arnés, está compuesta de dos especies, la primera más compacta, bien que flexible, ésta forma en su parte superior una especie de mortero, en cuya cavidad sale un bastago casi de la misma naturaleza y estructura. En la parte anterior é inferior tiene una Esponja de las que se conocen propiamente con este nombre, la que es producción del antecedente, aunque tan diferente en su estructura y organización. Esta está compuesta de diferentes celdillas, formadas por un tejido mole [blando], y consistente, en las que recibe el agua, llenándose con la mayor prontitud. De las de esta especie, y otras semejantes se sirven las gentes para enjugar el agua en diferentes casos». En efecto, el ejemplar conservado tiene en su interior un fragmento de estructura esponjosa de color pardo rojizo que originalmente sobresaldría como un filamento por el ósculo de la esponja mayor.
Estos ejemplares, algunos muy espectaculares y bellos, se asientan sobre peanas de madera imitando rocallas de estilo rococó con diferentes organismos adheridos como adorno, la mayoría moluscos bivalvos y gasterópodos, pequeños corales de las familias Faviidae y Caryophyllidae y crustáceos cirrípedos del género Balanus. En muchos casos, las colonias se han unido mediante clavos a soportes centrales de madera para dar firmeza a su colocación y favorecer su exhibición. La mayoría precisan de limpieza y, en mayor o menor grado, de restauración. Todas estas esponjas corresponden a especies propias del Caribe (Tabla 1).

Respecto a los ejemplares procedentes del Real Jardín Botánico, de los seis recogidos por Rojas Clemente, tres han sido determinados como Halichondria panicea, dos como Spongia officinalis y uno como Haliclona oculata. El ejemplar de la expedición Malaspina (1789-1794) está sin determinar (Tabla 1).

\section{FINALES DEL SIGLO XIX}

De este período se conservan unas 350 muestras de esponjas, más del $90 \%$ de ellas preparaciones microscópicas (Fig. 4), en la mayoría de las cuales están bien documentadas las técnicas de tinción empleadas. De estas preparaciones, 55 se han asignado a esta época con dudas, pues si bien carecen de datación, por sus características (tinción, forma de las etiquetas y ciertos datos numéricos anotados en éstas) son similares a otras datadas en dicho período. De los ejemplares conservados en alcohol (unos 40), casi todos fueron recogidos por González de Linares en las costas valencianas y Bretaña, estas últimas hacia 1883. En cuanto a las preparaciones, hay 88 de la Estación de Santander (más de 60 son de larvas, sin identificar) y ocho de Nápoles. Más de 160 se identificaron al nivel del género y otras 70 al de especie. Se contabilizan unas 40 especies, una de ellas, Cantabrina erecta, descrita por Ferrer-Hernández en 1914. Como dato curioso, uno de los ejemplares, procedente del litoral africano -sin localidad-, fue recogido por el explorador, médico y naturalista austríaco Emil Holub (1847-1902), que recorrió diversas regiones de África. La entrada se ha datado como anterior a 1894: en 2003 Van Soest y Erpenbeck lo determinaron como Smenospongia sp.

\section{LA ÉPOCA DE IGNACIO BOLIVAR (1901-1939)}

Algo más de la mitad de los ejemplares históricos de esponjas (un número ligeramente superior a 400) ingresaron en la época en que fue director Ignacio Bolívar (una veintena con dudas), cuando se creó el Laboratorio de Malacología y Animales Inferiores (Fig. 5). Casi un $25 \%$ son preparaciones microscópicas y el resto se conservan en alcohol. Se contabilizan 


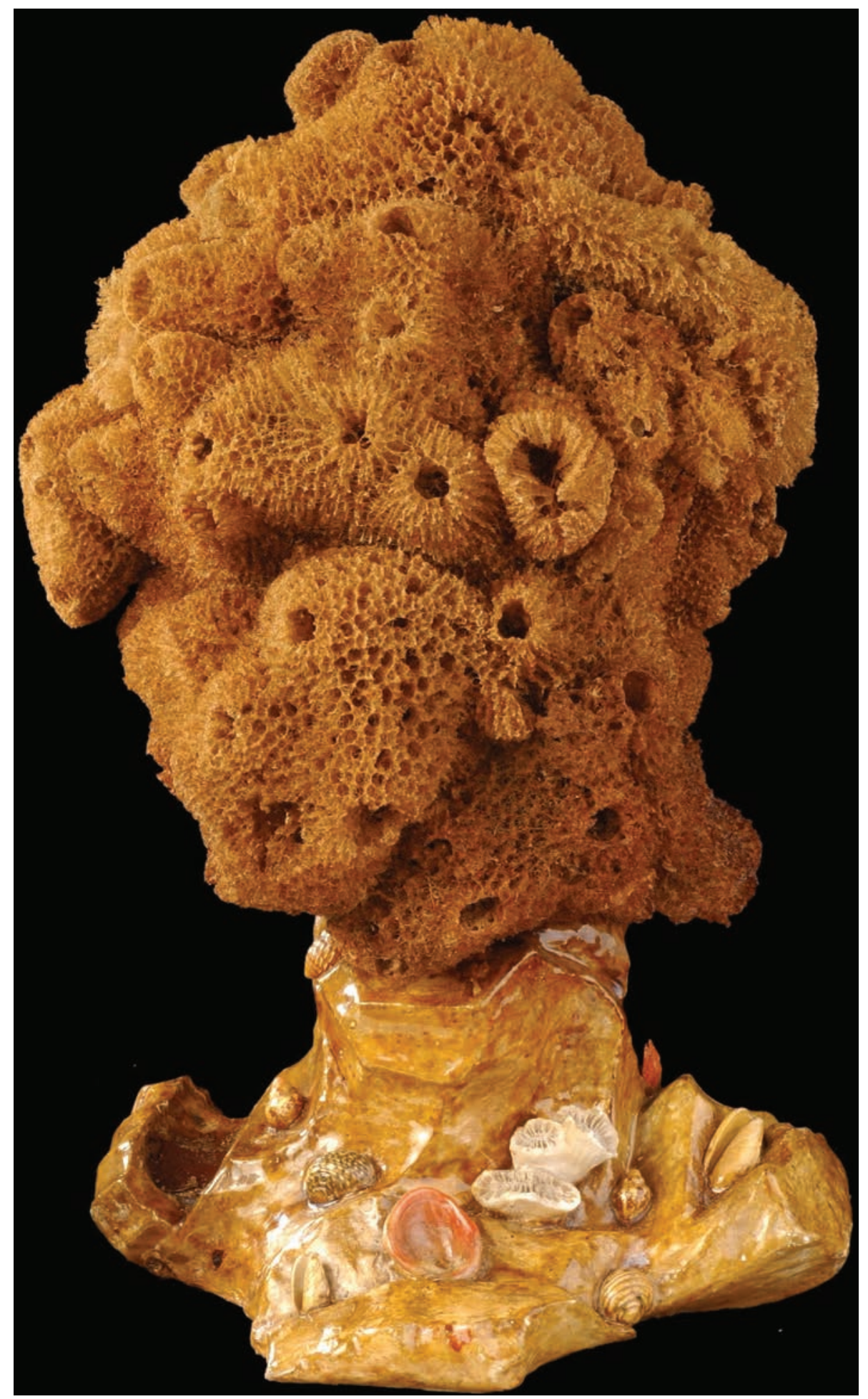

Fig. 3.- Smenospongia conulosa Pulitzer-Finali, 1986: MNCN 1.01/323. Ejemplar histórico de la colección Parra (Jesús Muñoz. Servicio de Fotografía del MNCN).

Fig. 3.- Smenospongia conulosa Pulitzer-Finali, 1986: MNCN 1.01/323. Historical sponges collection from Antonio Parra (Jesús Muñoz. MNCN Photography Service).

unas 120 especies, de unas 50 familias. Las más representadas son: Halichondridae, Geodiidae, Suberitidae, Axinellidae, Chalinidae y Grantiidae. Casi 140 ejemplares son del Cantábrico, la mayoría de Santander, unos 80 de las costas gallegas (sobre todo de Pontevedra), y más de 100 del Mediterráneo: 59 de Baleares (Mahón y Palma de Mallorca), casi una veintena del litoral valenciano y cinco de Málaga. Hay 14 procedentes de Nápoles, probablemente de la Estación de Biología, y cinco de Japón. 


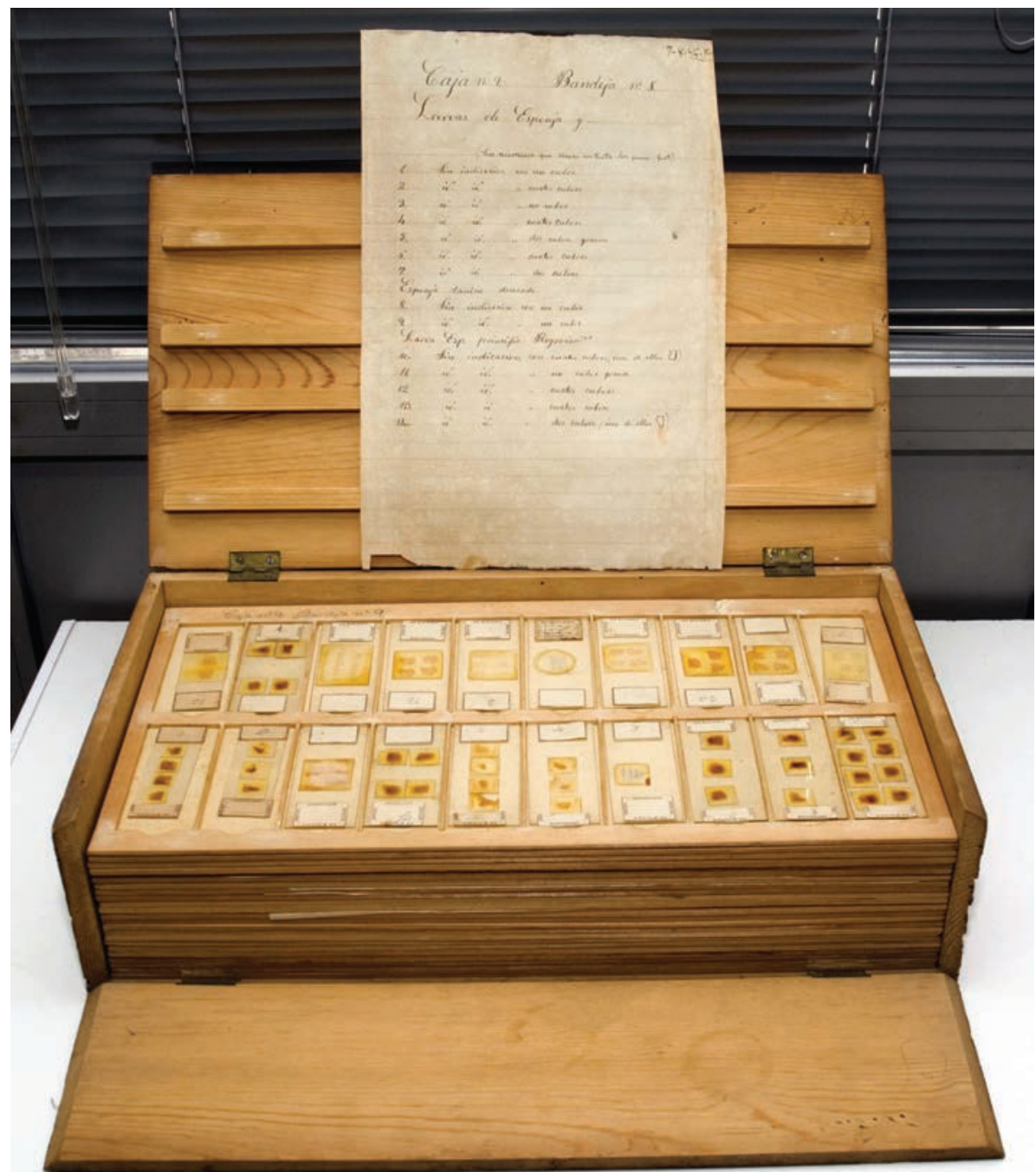

Fig. 4.- Preparaciones microscópicas de esponjas de finales del siglo XIX. La mayoría se montaron entre 1896 y 1897 (Fernando Señor. Servicio de Fotografía del MNCN).

Fig. 4.- Microscopic slides of sponges from the nineteenth century. Mostly were made between 1896 and 1897 (Fernando Señor. MNCN Photography Service).

En este período destacó la labor de Francisco Ferrer Hernández. Más de 280 ejemplares de la actual colección, de 46 familias y más de 100 especies, fueron identificados por él (Tabla 2). Otros 41 los colectó el propio Ferrer entre 1911 y 1917: 14 Calcarea, 23 Demospongiae y cuatro Hexactinellida. El 13 de marzo de 1915 donó al museo 111 preparaciones de esponjas.

Ferrer describió varias especies, de las que se conservan en la colección cuatro holotipos y tres sintipos. La primera descrita por él fue Scutastra cantabrica (Ferrer-Hernández, 1912), actualmente Erylus cantabricus (un sintipo MNCN 1.01/15). En este trabajo Ferrer hacía a modo de introducción una interesante exposición sobre los criterios que han servido para clasificar las esponjas, desde Gray en 1867 hasta los estudios de Minchin y Dendy, a finales del siglo XIX. Posteriormente describió varias especies más: Cantabrina erecta Ferrer-Hernández,
1914 (holotipo MNCN 1.01/12) y Siphonochalina balearica Ferrer-Hernández, 1916 (holotipo MNCN 1.01/14), demosponjas, y Vosmaeropsis gardineri Ferrer-Hernández, 1916 (holotipo MNCN 1.03/1) y Leucandra riojai Ferrer-Hernández, 1918 (holotipo MNCN 1.03/2), esponjas calcáreas. Además, se conservan sintipos de las especies: Leucandra sulcata Ferrer-Hernández, 1918 (MNCN 1.03/3) y Vosmaeropsis hispanica Ferrer-Hernández, 1933 (MNCN 1.03/24). Describió también otras especies nuevas: Leucandra bolivari Ferrer-Hernández, 1916 y Lissopocillon dendyi Ferrer-Hernández, 1916. La primera sigue considerándose como especie válida $\mathrm{y}$, aunque el autor mencionaba que el tipo se hallaba depositado en el museo, no se ha encontrado en la colección. La segunda, considerada en la actualidad como sinonimia de Phorbas ferrerhernandezi van Soest, 2002, está dedicada al ilustre espongiólogo español. Otras especies descritas por él cuyos tipos 


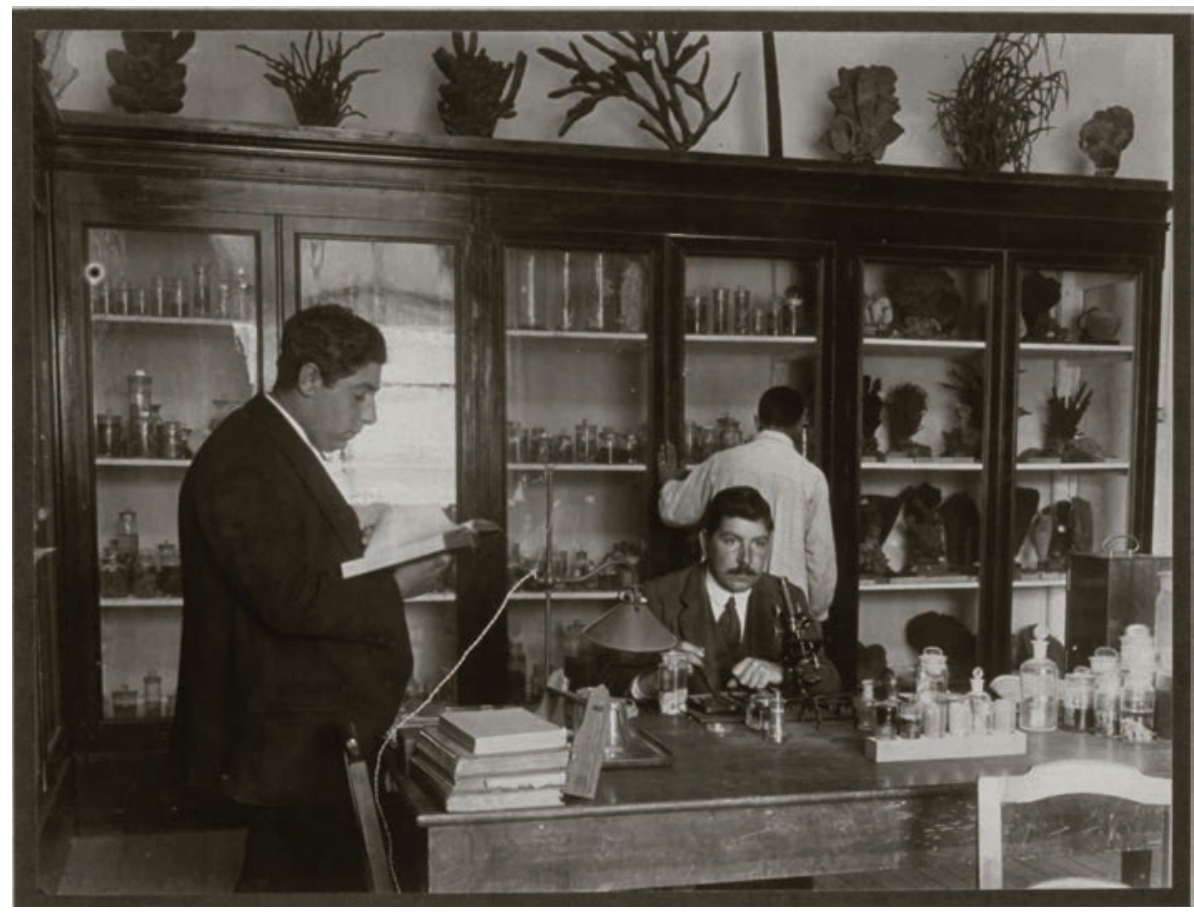

Fig. 5.- Laboratorio de Malacología y Animales Inferiores, creado en la etapa de Bolívar. Se aprecian en los armarios hasta 14 ejemplares de esponjas históricas, la mayoría de Parra (Archivo Fotográfico MNCN).

Fig. 5.- Laboratory of Malacología y Animales Inferiores, established in the Bolívar epoch. It can be seen 14 specimens of historical sponges in the cupboard, mostly from Parra collection (MNCN Photographic Archives).

Tabla 2.- Esponjas identificadas por Francisco Ferrer Hernández.

Table 2.- Sponges identified by Francisco Ferrer Hernández.

\begin{tabular}{llr}
\hline COLECCIÓN & FAMILIAS & ESPECIES \\
\hline CALCAREA & $\mathbf{6}$ & $\mathbf{1 7}$ \\
& Grantiidae & 7 \\
& Clathrinidae & 4 \\
& Heteropidae & 2 \\
& Sycettidae & 2 \\
& Leucosoleniidae & 1 \\
DEMOSPONGIAE & Leucaltidae & 1 \\
& $\mathbf{3 5}$ & $\mathbf{8 6}$ \\
& Chalinidae & 10 \\
& Geodiidae & 8 \\
& Axinellidae & 7 \\
& Halichondriidae & 5 \\
& Irciniidae/Suberitidae & 4 \\
& Ancorinidae/Pachastrellidae/Spongiidae/Tetillidae & 3 \\
& Callyspongidae/Dysideidae/Hymedesmiidae/Microcionidae/Mycalidae/Plakinidae/Raspaillidae/ & 2 \\
& Spongillidae/Theneidae & \\
HEXACTINELLIDA & Acarnidae/Bubaridae/Calthropellidae/Chondrosiidae/Clionidae/Darwinellidae/Desmacididae/ \\
& Dictyonellidae/Hamacanthidae/Hemiasterellidae/Myxillidae/Petrosiidae/Polymastidae/Tethyidae/ & 1 \\
& Thorectidae/Timeidae & $\mathbf{4}$
\end{tabular}

no se encuentran en la colección del MNCN son: Vosmaeropsis oruetai Ferrer-Hernández, 1918, Menanetia topsenti Ferrer-Hernández, 1918 actualmente adscrita al género Halichondria, y
Thorectandra diversispiculifera Ferrer-Hernández, 1918, en la actualidad considerada por el World Porifera Database (van Soest, 2010) con el status de "taxon inquirendum". 


\section{AGRADECIMIENTOS}

Al personal del Archivo y del Servicio Fotográfico del MNCN, María Ángeles Ramos (MNCN), Francisco Pando (Real Jardín Botánico) y Javier Cremades (Universidad de La Coruña). A las dos personas que han evaluado el artículo por sus valiosas observaciones, que han enriquecido este trabajo.

\section{Referencias}

Calatayud-Arinero, Ma . A., 1987. Catálogo de documentos del Real Gabinete de Historia Natural (1752-1786). CSIC. Madrid. 417 pp.

Cremades, J., 1990. El herbario de algas marinas de Simón de Rojas Clemente y Rubio (1777-1827). Tesis doctoral. Universidad de Santiago.

Cremades, J., 1993. Contribución al conocimiento de la obra ficológica de Simón de Rojas Clemente (1777-1827): tipificación de los nuevos nombres de su Ensayo. Anales del Jardín Botánico de Madrid, 51(1): 3-32.

Ferrer-Hernández, F., 1912. Notas sobre algunas esponjas de Santander. Boletín de la Real Sociedad Española de Historia Natural, 12: 573-589.

Ferrer-Hernández, F., 1914a. Estudios sobre espongiarios. Boletín de la Real Sociedad Española de Historia Natural, 14: 451-455.

Ferrer-Hernández, F., 1914b. Esponjas del Cantábrico. Parte Primera. I. Calcarea. II. Euceratosa. Trabajos del Museo Nacional de Ciencias Naturales. Serie Zoológica, 14: 3-36.

Ferrer-Hernández, F., 1914c. Esponjas del Cantábrico. Parte Segunda. III. Myxospongida. IV. Tetraxonida. V. Trizonida. Trabajos del Museo Nacional de Ciencias Naturales. Serie Zoológica, 17: 1-46.

Ferrer-Hernández, F., 1916. Fauna del Mediterráneo Occidental. Esponjas españolas. Trabajos del Museo Nacional de Ciencias Naturales. Serie Zoológica, 27: $1-52$.

Ferrer-Hernández, F., 1917. Algunas esponjas interesantes de Málaga. Boletín de la Real Sociedad Española de Historia Natural, 17: 228-230.

Ferrer-Hernández, F., 1918. Esponjas del litoral de Asturias. Trabajos del Museo Nacional de Ciencias Naturales. Serie Zoológica, 36: 1-39.

Ferrer-Hernández, F., 1919. Apuntes para la fauna ibérica. Boletín de la Real Sociedad Española de Historia Natural, 19: 197-201.
Ferrer-Hernández, F., 1921. Esponjas recogidas en la campaña preliminar del "Giralda". Boletín de Pescas, 6(59-60): 161-177.

Ferrer-Hernández, F., 1932a. Nota preliminar sobre una nueva esponja hexactinélida. Boletín de la Real Sociedad Española de Historia Natural, 32: 99-101.

Ferrer-Hernández, F., 1932b. Una nueva especie del género Acanthascus (Spong. Hexac. Hexasteropho.). Boletín de la Real Sociedad Española de Historia Natural, 32: 265-268.

Ferrer-Hernández, F., 1933. Sobre algunas esponjas de Marín (Galicia). Boletín de la Real Sociedad Española de Historia Natural; 33: 347-358.

Franco-Dávila, P., 1767. Catalogue Systématique et Raisonné des Curiosités de la Nature et de l'Art qui composent le Cabinet de Mr. Davila avec figures en taille douce de plusieurs morceaux qui n'avaient point encoré eté gravés. Briasson. París. xxxvi + 571 pp., 22 lám.

Parra, A., 1787. Descripción de diferentes piezas de Historia Natural las mas del ramo marítimo representadas en setenta y cinco láminas. Imprenta de la Capitanía General. La Habana. 195 + [5] pp., 75 lám.

Sánchez-Almazán, J. I. (Ed.). 2012. Pedro Franco Dávila (1711-1786). De Guayaquil a la Royal Society. La época y la obra de un ilustrado criollo. CSIC. Madrid. 352 pp.

van Soest, R., 2010. Thorectandra diversispiculifera FerrerHernandez, 1918. In: Van Soest, R. W. M., Boury-Esnault, N., Hooper, J. N. A., Rützler, K., de Voogd, N. J., Alvarez de Glasby, B., Hajdu, E., Pisera, A. B., Manconi, R., Schoenberg, C., Klautau, M., Picton, B., Kelly, M., Vacelet, J., Dohrmann, M., Díaz, M.-C., Cárdenas, P., Carballo, J. L. \& Rios Lopez, P., 2017. World Porifera database. http://www.marinespecies.org/porifera/porifera. php? $\mathrm{p}=$ taxdetails\&id=132453 [Consultada el 19.05.2017].

van Soest, R. W. M., Boury-Esnault, N., Hooper, J. N. A., Rützler, K., de Voogd, N. J., Alvarez de Glasby, B., Hajdu, E., Pisera, A. B., Manconi, R., Schoenberg, C., Klautau, M., Picton, B., Kelly, M., Vacelet, J., Dohrmann, M., Díaz, M.-C., Cárdenas, P., Carballo, J. L. \& Rios Lopez, P., 2017. World Porifera database. http://www. marinespecies.org/porifera [Consultada el 19.05.2017].

Villena, M., Sánchez-Almazán, J. I., Muñoz, J. \& Yagüe, F., 2009. El gabinete perdido. Pedro Franco Dávila y la historia natural del Siglo de las Luces. CSIC. Madrid. $1.170 \mathrm{pp}$.

WoRMS Editorial Board, 2017. World Register of Marine Species. http://www.marinespecies.org [Consultada el 19.05.2017]. https://dx.doi.org/10.14284/170 
Apéndice 1.- Listado de especies de las esponjas históricas del MNCN. Las letras indican, cuando el dato se conoce, los científicos que las determinaron. B: Boscá; E: Erpenbeck; ER: Enrique Rioja; FH: Ferrer Hernández; GL: González de Linares; JR: José Rioja; O: Orueta; OB: Odón de Buen; U: Uriz; VS: Van Soest. Se han actualizado los nombres científicos. A la derecha del nombre actual de la esponja aparece la denominación antigua.

Appendix 1.- List of species of historical sponges of MNCN. The letters indicate, when the information is known, the scientists who identified them (B: A. Boscá; E: D. Erpenbeck; ER: Enrique Rioja; FH: Ferrer Hernández; GL: González de Linares; JR: José Rioja; O: D. Orueta; OB: Odón de Buen; U: Uriz; VS: R.W.M. van Soest). Specific names have been updated. On the right of each name updated the old name is provided.

Aaptos aaptos (Schmidt, 1864) anteriormente Tuberella aaptos (Schmidt, 1864) (FH)

Acanthella acuta Schmidt, 1862 (U)

Acanthella erecta (Carter, 1876) anteriormente Bubaris vermiculata erecta (Carter, 1876) (FH)

Acarnus tortilis Topsent, 1892 (FH)

Adreus fascicularis (Bowerbank, 1866) (FH)

Agelas conifera (Schmidt, 1870) anteriormente Chalinopsis conifera (Schmidt, 1870) (E y VS)

Agelas dispar Duchassaing y Michelotti, 1864

Agelas sceptrum (Lamarck, 1815) (E y VS)

Aphroceras caespitosa (Haeckel, 1872) anteriormente Leuconia caespitosa (Haeckel, 1872) (FH)

Aphroceras corticata Lendenfeld, 1891 (FH)

Aplysilla sulfurea Schulze, 1878 (FH)

Aplysina aerophoba (Nardo, 1833) anteriormente Aplysina carnosa Schmidt, 1862

Aplysina archeri (Higgin, 1825) (E y VS)

Aplysina fistularis (Pallas, 1766) anteriormente Spongia fistularis Pallas, 1766

Aplysina lacunosa (Lamarck, 1814) (E y VS)

Arthuria canariensis (Miklucho-Maclay, 1868) anteriormente Leucosolenia canariensis (Miklucho-Maclay, 1868) (FH)

Ascandra falcata Haeckel, 1872 anteriormente Leucosolenia falcata Haeckel, 1872 (FH)

Asconema setubalense Kent, 1870 (E)

Axinella cannabina (Esper, 1794) (FH)

Axinella cinnamomea (Nardo, 1833) anteriormente Grantia cinnamomea Nardo, 1833 (FH)

Axinella dissimilis (Bowerbank, 1866) (FH)

Axinella polypoides Schmidt, 1862 (E y VS/FH/OB)

Axinella verrucosa (Esper, 1794)

Callyspongia fallax Duchassaing y Michelotti, 1864 (E y VS)

Callyspongia plicifera (Lamarck, 1814) (E y VS)

Callyspongia serpentina (Lamarck, 1814) (FH)

Callyspongia tenerrima Duchassaing y Michelotti, 1864 (E y VS)

Callyspongia vaginalis (Lamarck, 1814) (E y VS)

Calthropella stelligera (Schmidt, 1868) (FH)

Caminella intuta (Topsent, 1892) (FH)

Cantabrina erecta Ferrer Hernández, 1914 (MNCN 1.01/11; holotipo) (FH)

Chalinula limbata (Montagu, 1814) anteriormente Pachychalina limbata (Montagu, 1814) (FH)

Characella tripodaria (Schmidt, 1868) anteriormente Sphinctrella linaresi Ferrer-Hernandez, 1914 (FH)

Chondrosia reniformis Nardo, 1847 (FH)

Clathria compressa (Bowerbank, 1875)

Clathria coralloides (Scopoli, 1772) (ER)
Clathria lobata Vosmaer, 1880 (FH)

Clathria spinosa (Wilson, 1902) (E y VS)

Clathrina clathrus (Schmidt, 1864) (FH)

Clathrina coriacea (Montagu, 1814) anteriormente Leucosolenia coriacea (Montagu, 1814) (FH)

Clathrina reticulum (Schmidt, 1862) anteriormente Leucosolenia reticulum (Schmidt, 1862) (FH)

Cliona celata Grant, 1826 (E y VS/FH/GL/JR/O)

Cliona patera (Hardwicke, 1820) anteriormente Poterion neptuni Schlegel, 1858 (E y VS)

Craniella zetlandica (Carter, 1872)

Desmacidon fruticosum (Montagu, 1814) (ER/FH/GL)

Dictyonella marsilii (Topsent, 1893) anteriormente Stylotella marsilii Topsent, 1893 (FH)

Diplastrella bistellata (Schmidt, 1862) anteriormente Spirastrella bistellata (Schmidt, 1862) (FH)

Dragmacidon egregium (Ridley, 1881) anteriormente Axinella egregia (Ridley, 1881) (FH)

Dysidea fragilis (Montagu, 1814) (FH)

Dysidea pallescens (Schmidt, 1862)

Dysidea tupha (Martens, 1824) anteriormente Spongelia elegans Nardo, 1847 (ER/ FH)

Echinodictyumpennatum(DuchassaingyMichelotti, 1864)(EyVS)

Ephydatia fluviatilis (Linnaeus, 1759) (B/ ER)

Erylus cantabricus (Ferrer Hernández, 1912) (MNCN 1.01/15; sintipo) anteriormente Scutastra cantabrica Ferrer Hernández, 1912 (FH)

Erylus discophorus (Schmidt, 1862) (FH)

Euplectella aspergillum Owen, 1841

Eurypon lacazei (Topsent, 1891) anteriormente Echinodictyum lacazei Topsent, 1892 (FH)

Farrea occa Bowerbank, 1862 (FH)

Fasciospongia cavernosa (Schmidt, 1862) anteriormente Stelospongia aspergillum (Schmidt, 1868) (FH/U)

Gelliodes wilsoni Carballo, Aquilar-Camacho, Knapp \& Bell, 2013 anteriormente Gelliodes fibrosa (Wilson, 1925)

Geodia barretti Bowerbank, 1858

Geodia cydonium (Jameson, 1811) anteriormente Cydonium muelleri Fleming 1828 (FH)

Geodia gigas Schmidt, 1862 anteriormente Cydonium gigas (Schmidt, 1862)

Geodia megastrella Carter, 1876 (FH)

Grantia capillosa (Schmidt, 1862) anteriormente Sycon capillosus (Schmidt, 1862)

Grantia compressa (Fabricius, 1780) (FH)

Halichondria albescens Johnston, 1842 (FH)

Halichondria bowerbanki Burton, 1930 anteriormente Reniera bowerbanki (Bowerbank, 1882) (FH)

Halichondria panicea (Pallas, 1766) anteriormente Ophlitaspongia seriata (sensu Johnston, 1842) (FH)

Haliclona angulata (Bowerbank, 1866) (FH)

Haliclona cinerea (Grant, 1826) anteriormente Reniera permollis (Bowerbank, 1866) (FH)

Haliclona fibulata (Schmidt, 1862) anteriormente Gellius fibulatus (Schmidt, 1862) (FH)

Haliclona flagellifera (Ridley \& Dendy, 1886) anteriormente Gellius flagellifer Ridley \& Dendy, 1886

Haliclona indistincta (Bowerbank, 1866) anteriormente Reniera indistincta (Bowerbank, 1866) (FH)

Haliclona oculata (Pallas, 1766) anteriormente Chalina oculata (Linnaeus, 1759) (FH)

Haliclona rectangularis (Ridley \& Dendy, 1886) anteriormente Chalina rectangularis Ridley \& Dendy, 1886 (FH)

Haliclona rosea (Bowerbank, 1866) (FH) 
Haliclona simulans (Johnston, 1842) anteriormente Reniera densa (Bowerbank, 1866) (FH)

Hamacantha falcula (Bowerbank, 1874) (FH)

Hemimycale columella (Bowerbank, 1874) anteriormente Stylotella columella (Bowerbank, 1874) (FH)

Hippospongia lachne (de Laubenfels, 1936) (E y VS)

Hyalonema apertum Schulze, 1886 (FH)

Hyattella cavernosa (¿?) (Pallas, 1766) (E y VS)

Hymeniacidon caruncula Bowerbank, 1859 (FH)

Hymeniacidon perlevis (Montagu, 1814) (FH/GL/U)

Ircinia strobilina (Lamarck, 1816) (FH)

Ircinia variabilis (Schmidt, 1862) (FH)

Leucandra aspera (Schmidt, 1862) (FH)

Leucandra riojai Ferrer Hernández, 1918 (MNCN 1.03/2; holotipo) (FH)

Leucandra rodriguezi (Lackschewitz, 1886) (FH)

Leucandra sulcata Ferrer Hernández, 1918 (MNCN 1.03/3; sintipo) (FH)

Leucetta solida (Schmidt, 1862) anteriormente Leuconia solida (Schmidt, 1862)

Leucosolenia complicata (Montagu, 1814) (FH)

Mycale mirabilis (Lendenfeld, 1887) (E y VS)

Mycale syrinx (Schmidt, 1862) (FH/OB)

Mycale tunicata (Schmidt, 1862) (FH)

Myxilla rosacea (Lieberkühn, 1859) (FH)

Niphates erecta Duchassaing y Michelotti, 1864 (E y VS)

Ophlitaspongia papilla Bowerbank, 1866 (FH)

Oscarella lobularis (Schmidt, 1862) (FH)

Pachastrella ovisternata Lendenfeld, 1894 (FH)

Pachymatisma johnstonia (Bowerbank in Johnston, 1842) (FH/GL)

Penares euastrum (Schmidt, 1868) anteriormente Erylus euastrum (Schmidt, 1868) (FH)

Penares helleri (Schmidt, 1864) (FH)

Periphragella elisae Marshall, 1875 (FH)

Phakellia robusta Bowerbank, 1866 (FH)

Phakellia ventilabrum (Linnaeus, 1767) (FH)

Phorbas ferrerhernandezi van Soest, 2002 anteriormente Lissopocillon dendyi Ferrer-Hernandez, 1916 (E)

Phorbas fictitius (Bowerbank, 1866) anteriormente Plumohalichondria fictitia (Bowerbank, 1866) (FH)

Plakortis simplex Schulze, 1880 (FH)

Poecillastra compressa (Bowerbank, 1866) anteriormente Pachastrella compressa (Bowerbank, 1866) (FH)

Polymastia uberrima (Schmidt, 1870) (FH)

Raspailia howsei (Bowerbank, 1866) (FH)
Sarcotragus foetidus Schmidt, 1862 anteriormente Hircinia muscarum (Schmidt, 1864) (FH)

Sarcotragus spinosulus Schmidt, 1862 anteriormente Hircinia spinulosa Schmidt, 1862 (FH)

Semperella cucumis Schulze, 1895

Sericolophus reflexus (Ijima, 1894) anteriormente Corythophora ijimae Ferrer-Hernandez, 1932 (FH)

Siphonochalina balearica Ferrer Hernández, 1916 (MNCN 1.01/14; holotipo) (FH)

Siphonochalina coriacea Schmidt, 1868 (ER/FH)

Smenospongia conulosa Pulitzer-Finali, 1986 (E y VS)

Spongia conifera (Lendenfeld, 1886) anteriormente Euspongia trincomalensis Lendenfeld, 1889 (FH)

Spongia irregularis (Lendenfeld, 1889) anteriormente Euspongia irregularis Lendenfeld, 1889 (FH)

Spongia lignea Hyatt, 1877 anteriormente Euspongia officinalis var. dura Lendenfeld, 1889

Spongia officinalis Linnaeus, 1759 anteriormente Euspongia officinalis (Linnaeus, 1759) (FH)

Spongilla alba Carter, 1849 anteriormente Spongilla lacustris (Linnaeus, 1759) (FH)

Stelletta grubii Schmidt, 1862 anteriormente Stelletta grubei (Schmidt, 1862) (FH)

Stelletta simplicissima (Schmidt, 1868) (FH)

Stelligera stuposa (Ellis \& Solander, 1786) anteriormente Dictyocylindrus stuposus (Ellis \& Solander, 1786) (GL)

Stryphnus ponderosus (Bowerbank, 1866) anteriormente Stelletta aspera Carter, 1871 (FH)

Suberites carnosus (Johnston, 1842) (FH)

Suberites domuncula (Olivi, 1792) (ER/FH/GL)

Suberites ficus (Johnston, 1842) anteriormente Ficulina ficus (Johnston, 1842) (FH)

Sycon ciliatum (Fabricius, 1780) (GL)

Sycon elegans (Bowerbank, 1845)

Sycon raphanus Schmidt, 1862 (FH)

Thethya aurantium (Pallas, 1766)

Tethya lyncurium (Linnaeus, 1767) (FH/JR)

Tetilla truncata Topsent, 1890 (FH)

Thenea muricata (Bowerbank, 1858) (FH)

Thenea schmidti Sollas, 1886 (FH)

Vosmaeropsis gardineri Ferrer Hernández, 1916 (MNCN 1.03/1; holotipo) (FH)

Vosmaeropsis hispanica Ferrer Hernández, 1933 (MNCN 1.03/24; sintipo) (FH)

Xestospongia muta (Schmidt, 1870) (E y VS) 
Apéndice 2.- Carta de Ferrer del 23 de abril de 1932 a la Junta para la Ampliación de Estudios (JAE).

Appendix 2.- Letter from Ferrer, april 23th 1932, to JAE.

(Documento ACN0352/007/001, Archivo del MNCN)

«Mi labor como único especialista en esponjas que existe en España, ha de tener por objeto reunir y publicar cuantos datos pueda adquirir de las esponjas de nuestras costas; clasificar ejemplares recogidos o que pueda recoger en varias excursiones, estudiar su biología y afrontar problemas como los de regeneración, sexualidad, reproducción etc.

Por mis anteriores trabajos, bien apreciados de los especialistas extranjeros, se conoce bastante la fauna espongiológica del Cantábrico y un poco la de Baleares; mas es imprescindible la recolección de especies del Atlántico (costas gallegas y andaluzas) y de casi todo el litoral mediterráneo.

Para dedicarme a ello dispongo todos los días de las mañanas o las tardes y también de toda la jornada durante las épocas de vacaciones escolares.

Madrid 23 Abril 1932

Francisco Ferrer Hernández» 